\title{
Adaptación transcultural del cuestionario KIDSCREEN para medir calidad de vida relacionada con la salud en población mexicana de 8 a 18 años
}

Cross-cultural adaptation of the KIDSCREEN questionnaire to measure health-related quality of life in the 8 to 18 year-old Mexican population

Centro de Estudios Avanzados, Universidad de de Playa Ancha, Valparaíso Chile. Departamento de Cultura Arte y Desarrollo Humano, Centro

Universitário de Sur,

Universidad de Guadalajar Av. Enrique Arreola Silva 883, Colonia Centro. 49.000 Ciudad Guzmán Jalisco México.

carlosh@cusur.udg.mx ${ }^{2}$ Catalan Agency for Health Information, Assessment and Quality.

${ }^{3}$ Universidad de Santiago de Chile.
Abstract The scope of this study was to develop the culturally-adapted version of the KIDSCREEN-52 questionnaire for Mexican adolescents aged 8 to 18, and to analyze its reliability and validity. A translation and back translation was carried out from English to Spanish and vice versa. Cognitive interviews were conducted and confirmatory factor analysis (CFA) was used to verify the content validity of the questionnaire. Based on previous studies (i.e. the Argentinian version) mean scores of dimensions were compared to assess construct validity. Reliability was also analyzed. The results showed that the content validity of the version retained the original concepts, duly adapted to the Mexican culture and language. The AFC confirmed the structure of the 10 original dimensions. Two scales achieved the ceiling effect. The instrument's internal consistency was acceptable (ranged from 0.5 to 0.86 ), and reproducibility obtained low coefficients in 5 dimensions (0.22 to 0.78). The Mexican KIDSCREEN-52 version is equivalent to the original in terms of content, structure, and construct validity. The conclusion was that the KIDSCREEN52 tool is suitable for application in the Mexican population aged 8 to 18. Further studies are needed to explore its application in clinical settings.

Key words Children, Adolescents, Quality of life, Validation studies, Questionnaires, Mexico
Resumen Con el objeto de adaptar culturalmente el cuestionario KIDSCREEN-52 a adolescentes mexicanos de 8 a 18 años, y analizar su fiabilidad y validez, se realizó la traducción del cuestionario del inglés al español y una retro traducción. Se realizaron entrevistas cognitivas y un análisis factorial confirmatorio (AFC) para verificar la validez del contenido del cuestionario. Se compararon las puntuaciones de las dimensiones siguiendo los mismos criterios que en otras versiones (ej. Argentina) para analizar la validez de constructo, y se verificó la fiabilidad. La validez del contenido mantuvo las características del cuestionario original. El AFC confirmó la estructura de las 10 dimensiones originales. Dos escalas mostraron efecto techo. La consistencia interna del instrumento fue aceptable (rango 0,5-0,86) y la reproducibilidad obtuvo coeficientes bajos en 5 dimensiones $(0,22-0,78)$. La versión mexicana del cuestionario KIDSCREEN-52 es equivalente al original en términos de contenido, estructura, y validez de constructo. En conclusión, el instrumento KIDSCREEN-52 es adecuado para aplicarse en población mexicana de estudiantes de 8 a 18 años. Son necesarios nuevos estudios para explorar su aplicación en contextos clínicos.

Palabras clave Niños, Adolescentes, Calidad de vida, Estudios de validación, Cuestionarios, Méxi- 


\section{Introducción}

En las últimas décadas ha habido un interés creciente en la calidad de vida ${ }^{1,2}$ en cuanto a percepción, porque ha permitido comprender mejor la perspectiva de los pacientes y personas cuando toman decisiones. En el entorno de la enfermedad, su medición ha permitido entre otras cosas escoger entre tratamientos ${ }^{2}$, mientras que con población sana ha permitido ver por ejemplo la forma en que esta se asocia con problemas o riesgos a la salud. La población entre 10 y 19 años representa un $17.7 \%$ de la población mundial ${ }^{3}$. Aunque en esta edad las personas generalmente gozan de buena salud y algunas pueden ser incluso resilientes ante la adversidad, muchas son afectadas en su salud por las condiciones en que viven $^{3}$. La calidad de vida relacionada con la salud (CVRS), se ha definido como "la medida concentrada en el concepto de salud o los resultados en el campo de la salud"4 y también ha sido definida como "la salud física y mental percibida indivi-

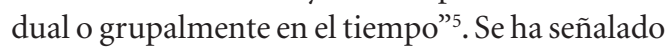
la utilidad que la medición de la CVRS tiene en la pediatría ${ }^{6}$ y el conocimiento de factores vinculados a la calidad de vida es aplicable en contextos donde hay adolescentes como servicios de salud, clínicas y hospitales al ver más allá del diagnóstico y observar a la persona y sus relaciones ${ }^{7}$. Además las medidas de CVRS han mostrado ser útiles para estudiar poblaciones de adolescentes sanos ${ }^{8-}$ ${ }^{11}$. Para que esto sea posible es fundamental que se cuente con instrumentos para poder valorarla. Una revisión de instrumentos de CVRS a nivel internacional en 2005 reportó que los instrumentos encontrados habían sido creados o adaptados en países europeos o anglosajones ${ }^{12}$ y aún en 2012 los instrumentos de CVRS en Iberoamérica era escasa ${ }^{13,14}$. Ante tal escasez, la adaptación transcultural aparece con grandes ventajas cuando ya se tienen instrumentos desarrollados, en comparación a la creación de nuevos ${ }^{15}$.

El Instrumento KIDSCREEN (Screening for and promotion of Health Related Quality of Life in Children and Adolescents), sirve para medir la CVRS, fue creado entre 13 países europeos con el propósito de desarrollar un instrumento multicultural que pudiera ser aplicado en todos los países participantes ${ }^{16,17}$. La versión de 52 ítems del instrumento, mide 10 dimensiones de calidad de vida relacionada con la salud: bienestar físico (5 ítems), bienestar psicológico (6 ítems), estado de ánimo (7 ítems), autopercepción (5 ítems), autonomía (5 ítems), relación con los padres y vida familiar (6 ítems), recursos económicos (3 ítems), amigos y apoyo social (6 ítems), entorno escolar (6 ítems), aceptación social (Bullying) (3 ítems). La versión de 27 ítems, formada de 5 dimensiones y la versión de 10 ítems permiten obtener un índice global. Las dimensiones fueron construidas y piloteadas usando la información de más de 3000 niños y adolescentes europeos. Se ejecutaron además análisis psicométricos, se aplicó la teoría de respuesta al ítem y modelos de ecuación estructural para determinar las características ópticas del ítem y la escala del cuestionario. Se analizó si los ítems mostraban un funcionamiento diferencial (DIF), lo que posibilitó la comparación de las dimensiones identificadas de calidad de vida a través de 13 países Europeos. El KIDSCREEN-52 fue enviado por correo a una muestra representativa por país de aproximadamente 1800 niños y sus padres (total $n=22,296$ ), que incluyó la muestra española ${ }^{16}$, y a partir de ello se produjeron los datos normativos. El análisis final de los instrumentos involucrando análisis nacionales y transculturales, confirmaron los resultados del estudio piloto. Las sub-escalas posibilitan la medida transcultural sobre el nivel de escala de intervalo asumiendo el modelo Rasch y resultando no DIF. El instrumento está disponible en varios lenguajes ${ }^{17,18} \mathrm{y}$ ha tenido procesos de adaptación transcultural en países Iberoamericanos: Argentina ${ }^{15}$, Brasil ${ }^{19}$, Chile ${ }^{20}$, Colombia $^{21}$, Portugal ${ }^{22}$. En México se inició el proceso de adaptación en febrero de 2011 y se aprobó la versión final mexicana del instrumento por el grupo KIDSCREEN en mayo de 2011.

Para el Scientific Advisory Comitee of the Medical Outcomes Trust (SAC), la adaptación cultural y del lenguaje se refiere a cuando el instrumento se adapta a culturas o lenguajes distintos al original. La traducción es considerada distinta a la adaptación del lenguaje y tiene dos pasos principales: la evaluación de la equivalencia conceptual y lingüística, y la evaluación de las propiedades de medida. La equivalencia conceptual se refiere a la equivalencia en relevancia y significado de los mismos conceptos en diferentes culturas y/o lenguajes. La equivalencia lingüística se refiere a la equivalencia de significado y redacción en la formulación de ítems, opciones de respuesta, y todos los aspectos del instrumento $\mathrm{y}$ sus aplicaciones ${ }^{23}$.

En México existe el interés de que los profesionales de la salud cuenten con instrumentos que permitan valorar la CVRS tanto niños como en adolescentes. Por esa razón nuestro objetivo fue obtener una versión Mexicana del cuestionario KIDSCREEN-52, adaptada para población 
mexicana de 8 a 18 años equivalente lingüísticamente a las demás versiones del KIDSCREEN, y analizar su fiabilidad y validez.

\section{Métodos}

\section{Diseño}

La adaptación lingüística del KIDSCREEN fue evaluada en el proceso de traducción y retro-traducción siguiendo los lineamientos del grupo KIDSCREEN $^{24}$ y recomendaciones previas ${ }^{23,25}$ (fase1) la adaptación cognitiva se realizó mediante entrevistas cognitivas con estudiantes ejecutadas acorde al manual para adaptación cultural del KIDSCREEN $^{24}$ (fase 2). La fiabilidad y análisis estructural, fue evaluada estadísticamente mediante datos de cuestionarios (fase 3) aplicados como parte del Observatorio de los riesgos y calidad de vida de estudiantes.

Participantes. En la fase 1, cuatro traductores calificados, la persona asignada por el grupo KIDSCREEN y los investigadores participaron en el proceso de traducción. En la fase 2 para las entrevistas cognitivas los participantes fueron una muestra a conveniencia no probabilística conformada de hombres ( $\mathrm{n}=5$, edad 9-17 años) y mujeres ( $\mathrm{n}=9$, edad 9-18 años). Para el análisis factorial confirmatorio (AFC) y la fase 3 , los cuestionarios se aplicaron a estudiantes desde tercer grado de primaria hasta primer semestre de universidad, de dos escuelas primarias (privada y pública), una secundaria privada y una universidad pública, en una ciudad con 100,000 habitantes entre agosto de 2011 y marzo de 2012 ( $\mathrm{n}=$ 1041). Para el análisis de reproducibilidad o consistencia externa los participantes fueron 32 estudiantes de una escuela primaria, una secundaria y una preparatoria con edad entre 10 a 18 años.

\section{Recolección de datos}

El proceso de adaptación fue realizado en tres fases: 1.-equivalencia lingüística, 2.-equivalencia conceptual y 3.-validez del constructo y fiabilidad.

Fase 1 Para lograr la equivalencia lingüística se realizó la traducción del KIDSCREEN 52 entre febrero y marzo de 2011. El objetivo de la traducción fue asegurar la equivalencia semántica y conceptual de la versión original y la versión traducida. Debido a que ya existía una versión del instrumento adaptada al castellano de España ${ }^{16}$, en la primera fase, dos lingüistas y una Doctora en Psicología la tradujeron por separado revisando la congruen- cia con la versión original en inglés. Los investigadores revisaron las traducciones en reuniones virtuales con los traductores. Esto produjo la primera versión mexicana del cuestionario KIDSCREEN 52. Esta versión fue retro-traducida por un tercer traductor independiente certificado en traducción español-inglés. Los investigadores y la responsable del Grupo Kidscreen de Alemania, compararon la versión retro-traducida con la versión original. Los comentarios generaron ajustes que se enfocaron en obtener equivalencia conceptual y lenguaje coloquial con apoyo del tercer traductor.

Fase 2 En Marzo de 2011 se realizaron las entrevistas cognitivas. El objetivo fue verificar la equivalencia conceptual ${ }^{25}$ de la versión mexicana del cuestionario KIDSCREEN 52 refinándolo para asegurar la aceptabilidad y comprensión ${ }^{24}$ de las instrucciones, preguntas y opciones de respuesta desde el punto de vista de los estudiantes. Para evaluar la validez de contenido ${ }^{25}$ que se refiere a las similitudes en relevancia y significado de los mismos conceptos medidos, se llevaron a cabo entrevistas con estudiantes $(\mathrm{n}=14)$ en tres rangos de edad (8-11, 12-15, 16-18) con el criterio de que hubiera hombres y mujeres en cada grupo.

Para las entrevistas, personal capacitado utilizaron un formato semi-estructurado en el que se les pidió a los estudiantes que contestaran el cuestionario. Al concluir se registró el tiempo invertido en la contestación. Después se utilizó el método "probing" ${ }^{24}$ se les preguntó sus dudas y para confirmar la comprensión del sentido de la pregunta se revisó ítem por ítem preguntándoles ¿por qué contesto esto? Cuando no se encontró congruencia entre la pregunta y la respuesta se le explicó la pregunta y se utilizó el método "paraphrasing" 24 solicitando que dijera como lo preguntaría él o ella con sus palabras. Al finalizar la entrevista se le preguntó cómo se sintió. Todo el material fue audio grabado. Las respuestas fueron documentadas y enviadas para su revisión por parte de los investigadores y después enviadas al representante alemán de KIDSCREEN a partir de los comentarios se realizaron los últimos ajustes con lo que se obtuvo la versión final de la versión mexicana del KISCREEN 52.

Para verificar si los ítems funcionaban de manera similar a los del instrumento origina ${ }^{25}$, se aplicó un análisis factorial confirmatorio (AFC). Este análisis fue realizado al interior del proyecto de la Universidad de Guadalajara: $\mathrm{Ob}$ servatorio de los comportamientos de riesgo y calidad de vida de estudiantes. Para el estudio se tomó la totalidad de los estudiantes de dos escuelas primarias $(\mathrm{n}=264)$ y una secundaria $(\mathrm{n}=99)$ y 
aquellos estudiantes que cumplían el criterio de tener un máximo de 18 años incorporados a primer semestre de dos generaciones de una Universidad $(\mathrm{n}=678)$ que representaba $65.1 \%$ del total de la población $(\mathrm{n}=1041)$ (Tabla 1). Los niños y adolescentes completaron el cuestionario en un espacio especial entre clases, los universitarios en la semana de inducción a la Universidad. Todos los estudiantes completaron los cuestionarios en su versión electrónica.

Fase 3 En esta fase se verificó la validez de constructo al comparar los estadísticos de cada escala entre el estudio actual y un estudio previo (Argentino) ${ }^{26}$. Para la comparación se calculó la media, desviación estándar y efecto piso techo.

En esta fase también se verificó la fiabilidad que es el grado en el cual el instrumento está libre de error aleatorio, estimando la consistencia interna que es la homogeneidad de los ítems que componen la escala, calculando los valores de alfa de Cronbach ( $n=1041)$, la consistencia externa o reproducibilidad, que es la estabilidad en el tiempo, se valoró mediante el coeficiente de correlación intraclase (CCI) con datos de 31 estudiantes de preparatoria (17 hombres y 15 mujeres $\mathrm{M}=$ 15.9 DE = 1.3) a quienes se les aplicó en dos ocasiones el instrumento con una diferencia de una semana entre aplicaciones. Los adolescentes completaron el cuestionario en versión electrónica en un espacio especial entre clases, dentro de un aula de clase con computadoras y con la presencia de un aplicador. Debido a la dificultad para aplicar a más grupos por segunda vez solo se hizo con los estudiantes señalados

La versión electrónica fue idéntica en apariencia al formato impreso y fue piloteada antes de la aplicación y existe evidencia de que versiones electrónicas del instrumento KIDSCREEN trabajan de manera similar a la versión en papel ${ }^{27}$.

\section{Consideraciones éticas}

El proyecto Observatorio de los comportamientos de riesgo y calidad de vida de estudiantes, del cual esta investigación forma parte, fue aprobado por el Comité de ética del Centro de Investigación en Comportamiento Alimentario (CICAN) de la Universidad de Guadalajara que analizó el proyecto y documentos anexos a la luz de los postulados de la Declaración de Helsinki, de las Pautas Éticas Internacionales para la Investigación Biomédica en Seres Humanos CIOMS 2002. Se pidió consentimiento informado de los y las estudiantes y de los padres de los y las menores de 18 años. La información fue confidencial. Se firmó la colaboración para uso del instrumento KIDSCREEN y su proceso de traducción y adaptación, el 15 febrero de 2011.

Tabla 1. Frecuencia de género, nivel educativo de la madre y del padre por grupos de edad y en toda la muestra.

\begin{tabular}{|c|c|c|c|c|c|c|}
\hline \multirow{2}{*}{$\begin{array}{c}\text { Variables } \\
\text { sociodemográficas }\end{array}$} & \multicolumn{2}{|c|}{ Niños } & \multicolumn{2}{|c|}{ Adolescentes } & \multicolumn{2}{|c|}{ Total } \\
\hline & $\mathbf{n}$ & $\%$ & $\mathbf{n}$ & $\%$ & $\mathbf{n}$ & $\%$ \\
\hline \multicolumn{7}{|l|}{ Género } \\
\hline Hombres & 96 & $46,4 \%$ & 340 & $40,8 \%$ & 436 & $41,9 \%$ \\
\hline Mujeres & 111 & $53,6 \%$ & 494 & $59,2 \%$ & 605 & $58,1 \%$ \\
\hline Total & 207 & $100,0 \%$ & 834 & $100,0 \%$ & 1.041 & $100,0 \%$ \\
\hline \multicolumn{7}{|l|}{ Nivel de estudios de la madre ${ }^{* * *}$} \\
\hline No fue a la escuela & 2 & $1,0 \%$ & 6 &, $8 \%$ & 8 & ,9\% \\
\hline Primaria & 18 & $9,0 \%$ & 156 & $21,5 \%$ & 174 & $18,8 \%$ \\
\hline Secundaria & 35 & $17,4 \%$ & 160 & $22,1 \%$ & 195 & $21,1 \%$ \\
\hline Preparatoria o Universidad & 146 & $72,6 \%$ & 403 & $55,6 \%$ & 549 & $59,3 \%$ \\
\hline Total & 201 & $100,0 \%$ & 725 & $100,0 \%$ & 926 & $100,0 \%$ \\
\hline \multicolumn{7}{|l|}{ Nivel de estudios del padre ${ }^{* * *}$} \\
\hline No fue a la escuela & 2 & $1,3 \%$ & 5 &, $7 \%$ & 7 &, $8 \%$ \\
\hline Primaria & 16 & $10,2 \%$ & 160 & $21,1 \%$ & 176 & $19,2 \%$ \\
\hline Secundaria & 22 & $14,0 \%$ & 225 & $29,7 \%$ & 247 & $27,0 \%$ \\
\hline Preparatoria o Universidad & 117 & $74,5 \%$ & 368 & $48,5 \%$ & 485 & $53,0 \%$ \\
\hline Total & 157 & $100,0 \%$ & 758 & $100,0 \%$ & 915 & $100,0 \%$ \\
\hline
\end{tabular}

Prueba de chi cuadrada para las diferencias de frecuencias entre grupos de edad: ${ }^{* * *} \mathrm{p}<0.001$

Los totales de nivel de estudios no suman 1041 debido a estudiantes que no reportaron el dato por desconocerlo o porque no tenían al progenitor. Grupos: Niños (8-11 años) adolescentes (12-18 años) 


\section{Análisis estadísticos}

Para la fase 1 , los datos cualitativos recuperados en las entrevistas cognitivas fueron analizados para identificar problemas en la comprensión y contestación de las instrucciones, preguntas, opciones de respuesta y conceptos de la versión mexicana del KIDSCREEN 52. Los datos recuperados por el cuestionario fueron analizados a partir de las instrucciones del grupo KIDSCREEN.

Para la fase 2, la estructura multidimensional del instrumento fue evaluada mediante Análisis Factorial Confirmatorio (AFC) sobre la matriz de correlaciones policorica. Para el análisis de la bondad de ajuste del modelo se utilizaron los indicadores más comunes como: (a) la raíz cuadrada media del error de aproximación root mean square error of aproximation (RMSEA), representa el nivel de discrepancia entre el modelo y los datos en la población, menor a .06 indica ajuste satisfactorio del modelo de acuerdo a Bentler y Bonett (1980) y Bollen (1986),(b) el índice de ajuste normalizado, de normed fit index (NFI); criterio mínimo mayor a 0,9 (c) los índices de ajuste comparativo comparative fit index (CFI) sobre .9 que puede interpretarse como un índice capaz de explicar el $90 \%$ de la relación (covariación) entre las variables medidas (d) la bondad de ajuste con ji cuadrado Chi square godness of-fit statistic.

Para la fase 3, el efecto piso se calculó como la proporción de participantes que contestaron en todos los ítems de un dominio, la categoría más baja y por el contrario el efecto techo, como la proporción de participantes que contestaron en todos los ítems de un dominio, la categoría más alta, se consideró aceptable un valor de hasta $15 \%{ }^{28}$. En la evaluación de la fiabilidad en la consistencia interna se utilizó el alfa de Cronbach, considerando como adecuado un coeficiente de $.70^{23}$, y para la consistencia externa o reproducibilidad el coeficiente de correlación intraclase (CCI) entre dos aplicaciones, considerando adecuado $.70^{23}$. Se computaron las puntuaciones medias de las dimensiones del KIDSCREEN-52 estandarizadas a una media de 50 y 1 desvío estándar de 10, de acuerdo a los datos de la versión Europea $^{23}$. Para la validez de constructo se calculó media, desviación estándar y se estimó el tamaño del efecto mediante el cálculo de la diferencia de medias estandarizadas (effect size) mediante el software $G^{\star}$ Power 3.1.6. ${ }^{29}$.

Para el análisis estadístico se usó el programa LISREL 8.50 (Jöreskog and Sórbom) (Scientific Software International, Inc. Illinois, USA), y el software estadístico Statistical Package for the Social Sciences (SPSS) V20 (SPSS Inc., Chicago, IL, USA).

\section{Resultados}

Para la fase 1, en la etapa de traducción directa hubo diferencias entre los traductores en $10 \mathrm{de}$ los 52 ítems. En la etapa de reconciliación, 3 de los ítems tuvieron acuerdo total y 7 solo entre un traductor y los investigadores. Después de la traducción inversa el responsable de la versión española del KIDSCREEEN y coautor de este documento (LR), realizó comentarios en 3 ítems e instrucciones y la responsable en el Grupo KIDSCREEN de Alemania hizo comentarios en 12 preguntas, las instrucciones y opciones de respuesta. Después de ello cinco ítems críticos requirieron una nueva traducción al inglés para confirmar su equivalencia. En el Cuadro 1 se comparan algunos ítems de la versión mexicana final con las versiones europea y española así como con la versión retrotraducida.

Para la fase 2, los participantes en las entrevistas cognitivas $(\mathrm{n}=14)$ fueron homogéneos respecto a etnia y lenguaje. Las edades fueron entre 8 a 17 años de edad. Durante las entrevistas cognitivas hubo comentarios relevantes en 8 ítems. Del grupo de 8 a 11 años se hicieron 8 comentarios, del grupo de 12 a 15 se hicieron 4 comentarios y del grupo de 16 a 18 años 7 comentarios. Los 8 ítems fueron discutidos en etapas desde los autores, CH y LR hasta la responsable en Alemania quien hizo 8 comentarios que se consideraron para la versión final (Cuadro 2). Las entrevistas cognitivas mostraron que los participantes comprendieron con facilidad los conceptos, preguntas e instrucciones. La mayoría de los respondientes completó el cuestionario entre 9 y 15 minutos.

Para el AFC participaron en el estudio 621 hombres (58\%) y 451 mujeres (42\%) entre 8 y 18 años, homogéneos respecto a etnia y lenguaje En el análisis de la estructura multidimensional del cuestionario, el AFC basados sobre una estimación WLSM (weighted least square estimator) muestran un buen ajuste para la solución de 10 dimensiones del cuestionario, los índices obtenidos fueron $\chi^{2}(1229)=8006.96, \mathrm{NFI}=0.97, \mathrm{RM}-$ $\mathrm{SEA}=0.48$ y CFI $=.98$; la carga factorial () entre el ítem y la dimensión resultaron óptimas, con errores estándar en todos los ítems de .01.

En la fase 3 la versión mexicana del KIDSCREEN 52 mostró en términos de validez de constructo alta equivalencia comparado con los su- 


\begin{tabular}{|c|c|c|c|c|}
\hline \multirow[t]{11}{*}{ 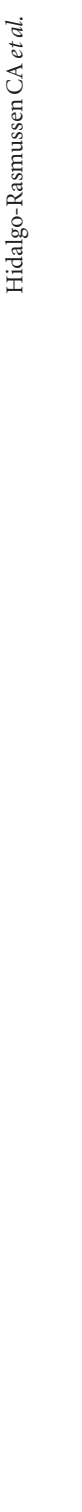 } & $\begin{array}{l}\text { Cuadro 1. Comparación } \\
\text { y versión mexicana fina }\end{array}$ & $\begin{array}{l}\text { de las versiones europea, es } \\
\text { l. Selección de instrucciones }\end{array}$ & $\begin{array}{l}\text { pañola, retrotraducción de l } \\
\text { e ítems. }\end{array}$ & a primera versión mexicana \\
\hline & $\begin{array}{l}\text { Versión original } \\
\text { europea }\end{array}$ & $\begin{array}{l}\text { Versión } \\
\text { española }\end{array}$ & $\begin{array}{l}\text { Retrotraducción de la } \\
\text { versión mexicana }\end{array}$ & $\begin{array}{c}\text { Versión } \\
\text { mexicana final }\end{array}$ \\
\hline & $\begin{array}{l}\text { When you think of } \\
\text { your answer please try } \\
\text { to remember the last } \\
\text { week. }\end{array}$ & $\begin{array}{l}\text { Cuando pienses en tu } \\
\text { respuesta, por favor } \\
\text { intenta recordar la última } \\
\text { semana, es decir, los } \\
\text { últimos siete días. }\end{array}$ & $\begin{array}{l}\text { When you think about } \\
\text { your answer, please try to } \\
\text { remember the last week, } \\
\text { i.e. the last seven days. }\end{array}$ & $\begin{array}{l}\text { Cuando pienses en tu } \\
\text { respuesta, por favor } \\
\text { intenta recordar la } \\
\text { última semana, es decir, } \\
\text { los últimos siete días. }\end{array}$ \\
\hline & $\begin{array}{l}\text { In general, how would } \\
\text { you say your health is? }\end{array}$ & $\begin{array}{l}\text { En general, ¿cómo dirías } \\
\text { que es tu salud? }\end{array}$ & $\begin{array}{l}\text { In general, how would } \\
\text { you say your health is? }\end{array}$ & $\begin{array}{l}\text { En general, ¿cómo dirías } \\
\text { que es tu salud? }\end{array}$ \\
\hline & $\begin{array}{l}\text { Have you felt satisfied } \\
\text { with your life? }\end{array}$ & $\begin{array}{l}\text { ¿Te has sentido satisfecho/ } \\
\text { a con tu vida? }\end{array}$ & $\begin{array}{l}\text { Have you felt satisfied } \\
\text { with your life? }\end{array}$ & $\begin{array}{l}\text { ¿Te has sentido } \\
\text { satisfecho/a con tu vida? }\end{array}$ \\
\hline & Have you felt lonely? & ¿Te has sentido solo/a? & Have you felt alone? & ¿Te has sentido solo/a? \\
\hline & $\begin{array}{l}\text { Have you felt under } \\
\text { pressure? }\end{array}$ & $\begin{array}{l}\text { ¿Te has sentido bajo } \\
\text { presión? }\end{array}$ & $\begin{array}{l}\text { Have you felt very } \\
\text { pressured/under pressure? }\end{array}$ & $\begin{array}{l}\text { ¿Te has sentido bajo } \\
\text { presión? }\end{array}$ \\
\hline & $\begin{array}{l}\text { Have your parent }(s) \\
\text { treated you fairly? }\end{array}$ & $\begin{array}{l}\text { ¿Tus padres te han tratado } \\
\text { de forma justa? }\end{array}$ & $\begin{array}{l}\text { Have your parents treated } \\
\text { you fairly? }\end{array}$ & $\begin{array}{l}\text { ¿Tus padres te han } \\
\text { tratado de forma justa? }\end{array}$ \\
\hline & $\begin{array}{l}\text { Have you spent time } \\
\text { with your friends? }\end{array}$ & $\begin{array}{l}\text { ¿Has pasado tiempo con } \\
\text { tus amigos/as? }\end{array}$ & $\begin{array}{l}\text { Have you spent time with } \\
\text { your friends? }\end{array}$ & $\begin{array}{l}\text { ¿Has pasado tiempo con } \\
\text { tus amigos/as? }\end{array}$ \\
\hline & $\begin{array}{l}\text { Have you and your } \\
\text { friends helped each } \\
\text { other? }\end{array}$ & $\begin{array}{l}\text { ¿Tú y tus amigos/as os } \\
\text { habéis ayudado unos/as a } \\
\text { otros/as? }\end{array}$ & $\begin{array}{l}\text { Have you and your } \\
\text { friends helped each other? }\end{array}$ & $\begin{array}{l}\text { ¿Tú y tus amigos/as se } \\
\text { han ayudado unos/as a } \\
\text { otros/as? }\end{array}$ \\
\hline & $\begin{array}{l}\text { Have other girls and } \\
\text { boys bullied you? }\end{array}$ & $\begin{array}{l}\text { ¿Te han intimidado o } \\
\text { amenazado otros chicos/ } \\
\text { as? }\end{array}$ & $\begin{array}{l}\text { Have other kids } \\
\text { intimidated or threatened } \\
\text { you? }\end{array}$ & $\begin{array}{l}\text { ¿Te han intimidado o } \\
\text { amenazado otros chicos/ } \\
\text { as? }\end{array}$ \\
\hline
\end{tabular}

puestos asumidos en la versión argentina ${ }^{26}$. Las puntuaciones medias del KIDSCREEN (Tabla 2) confirmaron las diferencias esperadas según sexo y edad. Las mujeres presentaron puntuaciones inferiores a las de los hombres en seis de las dimensiones con tamaño de efecto moderado en bienestar físico $(\mathrm{TE}=0.6)$ y mínimo en bienestar psicológico, estado de ánimo, autopercepción, autonomía y recursos económicos. $(\mathrm{TE}=0.2$ 0.3). Los niños y niñas tuvieron mejor CVRS que los adolescentes y las adolescentes de manera relevante en bienestar físico ( $\mathrm{TE}=0.8$ ), medio en autopercepción $(\mathrm{TE}=0.5)$ y tamaño de efecto mínimo ( $\mathrm{TE}=0.2-0.4$ ) en bienestar psicológico, autonomía, amigos y apoyo social, entorno escolar, aceptación social (Bullying), y recursos económicos mientras que fue mejor en adolescentes que en niños y niñas en aceptación social (Bullying) con un tamaño de efecto mínimo ( $\mathrm{TE}=$
0.4). El dominio entorno escolar en adolescentes fue el que obtuvo la menor puntuación. Tanto los valores del efecto piso como techo fueron adecuados y únicamente las dimensiones de autonomía y aceptación social (Bullying) obtuvieron proporciones superiores a $15 \%$.

La fiabilidad obtenida para las escalas fue adecuada (Alfa de Cronbach $\alpha_{\text {min }}=.51 \alpha_{\text {max }}=$ .86) aunque algo baja en tres dimensiones y su reproducibilidad mediante $\mathrm{CCI}\left(\mathrm{CCI}_{\min }=.47\right.$ $\left.\mathrm{CCI}_{\max }=.84\right)$, algo baja en ocho dimensiones. (Tabla 3)

\section{Discusión}

Fase 1. La validez del contenido para la versión mexicana del KIDSCREEN-52 en estudiantes con una edad entre 8 y 18 años, de educación básica a 


\begin{tabular}{|c|c|c|c|}
\hline $\begin{array}{l}N^{\circ} \text { de } \\
\text { ítem }\end{array}$ & Pregunta & $\begin{array}{l}\text { Dificultades/ } \\
\text { dudas }\end{array}$ & $\begin{array}{c}\text { Nueva } \\
\text { formulación }\end{array}$ \\
\hline 4 & ¿Has podido correr bien? & $\begin{array}{l}\text { Dudas en si "podido correr" se refería a } \\
\text { alguna dificultad para hacerlo }\end{array}$ & ¿Has podido correr bien? \\
\hline 8 & $\begin{array}{l}\text { ¿Te has sentido satisfecho/ } \\
\text { a con tu vida? }\end{array}$ & $\begin{array}{l}\text { Dificultades para distinguir "satisfecho" } \\
\text { de otras palabras }\end{array}$ & $\begin{array}{l}\text { ¿Te has sentido } \\
\text { satisfecho/a con tu vida? }\end{array}$ \\
\hline 16 & ¿Te has sentido harto/a? & $\begin{array}{l}\text { Como harto se podía entender en el } \\
\text { sentido de comer, sugirieron enfadado }\end{array}$ & $\begin{array}{l}\text { ¿Te has sentido } \\
\text { enfadado/a? }\end{array}$ \\
\hline 20 & $\begin{array}{l}\text { ¿Has estado contento/a } \\
\text { con tu ropa? }\end{array}$ & $\begin{array}{l}\text { Duda si se refería a una ropa o toda la } \\
\text { ropa que tenía }\end{array}$ & $\begin{array}{l}\text { ¿Has estado contento } \\
\text { por la ropa que tienes? }\end{array}$ \\
\hline 21 & $\begin{array}{l}\text { ¿Te ha preocupado tu } \\
\text { aspecto? }\end{array}$ & Dudas en la comprensión de "aspecto" & $\begin{array}{l}\text { ¿Has estado preocupado } \\
\text { por tu aspecto físico? }\end{array}$ \\
\hline 37 & $\begin{array}{l}\text { ¿Tienes suficiente dinero } \\
\text { para hacer cosas con tus } \\
\text { amigos/as? }\end{array}$ & $\begin{array}{l}\text { La palabra "cosas" podía tener varios } \\
\text { significados }\end{array}$ & $\begin{array}{l}¿ \text { Tienes suficiente dinero } \\
\text { para hacer actividades } \\
\text { con tus amigos/as? }\end{array}$ \\
\hline 39 & $\begin{array}{l}\text { ¿Has hecho cosas con } \\
\text { otros chicos/as? }\end{array}$ & La palabra "cosas" era ambigua & $\begin{array}{l}\text { ¿Has hecho actividades } \\
\text { con otros chicos/as? }\end{array}$ \\
\hline 43 & $\begin{array}{l}\text { ¿Has podido fiarte de tus } \\
\text { amigos/as? }\end{array}$ & Duda en la palabra "fiarte" & $\begin{array}{l}\text { ¿Has podido confiar en } \\
\text { tus amigos/as? }\end{array}$ \\
\hline
\end{tabular}

superior de escuelas públicas y privadas de México, fue alta.

Fase 2. Las entrevistas cognitivas confirmaron que el proceso de traducción-retrotraducción fue exitoso para desarrollar una versión mexicana del KIDSCREEN-52. Las entrevistas cognitivas también confirmaron que los conceptos traducidos, constructos, preguntas, instrucciones y alternativas de respuesta fueron entendidas de manera similar a la forma que los creadores del instrumento original intentaron, aún cuando hubo algunas diferencias semánticas entre el KIDSCREEN original y la versión mexicana. Las entrevistas cognitivas indicaron un alto grado de equivalencia entre la versión original y la versión traducida del KIDSCREEN-52. La versión mexicana del KIDSCREEN fue clara y fácil de entender y completar. No hubo ítems perdidos en las etapas de entrevistas cognitivas prueba piloto y aplicación final.

Fase 3. La validez de constructo de la versión mexicana parece ser alta. Hubo una alta equivalencia entre los resultados de la versión mexicana y la versión original.
Este estudio confirma investigaciones previas referidas a la validez y fiabilidad del KIDSCREEN52. Los valores de alfa en el estudio actual fueron altos en la mayoría de los dominios, excepto tres dimensiones que obtuvieron niveles aceptables, también observado en algunas dimensiones de otras versiones ${ }^{15,20}$. Los resultados del actual estudio confirman previos ${ }^{15-17,19,21,25,29}$.

Únicamente las dimensiones de autonomía y aceptación social (Bullying) superaron la proporción aceptable del efecto techo, hallazgo que ya fue reportado en la versión Argentina ${ }^{26}$.

Algunas dimensiones presentaron valores del test-retest ligeramente sub óptimos, especialmente la dimensión Ambiente escolar. Sin embargo este hecho ya ha sido descrito tanto en la versión original del instrumento como en otras investigaciones. En el estudio presente, además, este hecho puede haber sido agravado por el tamaño de muestra reducido con el que se analizó la reproducibilidad $^{17,18}$.

La validez de constructo del KIDSCREEN-52 ha sido probada en estudios previos al comparar pruebas resultantes entre muestras de personas 


\begin{tabular}{|c|c|c|c|c|c|c|}
\hline \multirow{25}{*}{ 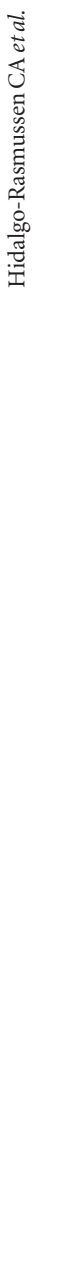 } & $\begin{array}{l}\text { Tabla 2. Puntuaciones promedio y desvi } \\
\text { edad y género }(\mathrm{N}=1041) \text {. }\end{array}$ & stándar d & e las di & nes del KI & DSCRE & or grupos de \\
\hline & \multirow{2}{*}{ Dimensiones CVRS ${ }^{*}$} & \multicolumn{2}{|c|}{ Niños/as } & \multicolumn{2}{|c|}{ Adolescentes } & \multirow{2}{*}{$\begin{array}{l}\text { Tamaño del } \\
\text { efecto }(d)\end{array}$} \\
\hline & & Media & $\mathrm{DE}$ & Media & $\mathrm{DE}$ & \\
\hline & Bienestar físico & 48.1 & 7.3 & 42.6 & 6.6 & 0.8 \\
\hline & Bienestar psicológico & 54.0 & 8.9 & 51.2 & 8.7 & 0.3 \\
\hline & Estado de ánimo & 45.8 & 8.8 & 44.8 & 7.5 & 0.1 \\
\hline & Autopercepción & 51.5 & 8.9 & 47.3 & 7.6 & 0.5 \\
\hline & Autonomía & 48.4 & 9.7 & 46.6 & 9.4 & 0.2 \\
\hline & Relación con los padres y vida familiar & 51.5 & 10.0 & 51.0 & 9.4 & 0.1 \\
\hline & Amigos y apoyo social & 50.8 & 10.2 & 48.6 & 9.4 & 0.2 \\
\hline & Recursos económicos & 46.4 & 8.5 & 44.7 & 7.3 & 0.2 \\
\hline & Entorno escolar & 57.1 & 9.6 & 53.3 & 7.9 & 0.4 \\
\hline & Aceptación social (Bullying) & 42.8 & 10.6 & 46.3 & 9.6 & 0.3 \\
\hline & \multirow{2}{*}{ Dimensiones CVRS } & \multicolumn{2}{|c|}{ Hombres } & \multicolumn{2}{|c|}{ Muheres } & Tamaño del \\
\hline & & Media & $\mathrm{DE}$ & Media & $\mathrm{DE}$ & efecto $(d)$ \\
\hline & Bienestar físico & 46.2 & 7.0 & 41.8 & 6.6 & 0.6 \\
\hline & Bienestar psicológico & 53.2 & 8.9 & 50.8 & 8.7 & 0.3 \\
\hline & Estado de ánimo & 46.1 & 7.8 & 44.2 & 7.6 & 0.2 \\
\hline & Autopercepción & 49.5 & 8.1 & 47.2 & 7.8 & 0.3 \\
\hline & Autonomía & 48.2 & 9.8 & 46.0 & 9.2 & 0.2 \\
\hline & Relación con los padres y vida familiar & 51.5 & 9.0 & 50.8 & 9.8 & 0.1 \\
\hline & Amigos y apoyo social & 49.3 & 9.4 & 48.8 & 9.7 & 0.1 \\
\hline & Recursos económicos & 46.0 & 7.7 & 44.4 & 7.4 & 0.2 \\
\hline & Entorno escolar & 53.9 & 8.6 & 54.2 & 8.3 & 0.0 \\
\hline & Aceptación social (Bullying) & 45.5 & 10.2 & 45.7 & 9.7 & 0.0 \\
\hline
\end{tabular}

${ }^{*}$ T-internacional RASCH.

Tabla 3. Propiedades métricas de la versión mexicana del KIDSCREEN-52 (N=1041).

\begin{tabular}{lccccc}
\hline \multicolumn{1}{c}{$\begin{array}{c}\text { Dimensiones } \\
\text { CVRS }^{*}\end{array}$} & $\begin{array}{c}\text { Número } \\
\text { de ítems }\end{array}$ & $\begin{array}{r}\text { Efecto } \\
\text { piso (\%) }\end{array}$ & $\begin{array}{c}\text { Efecto } \\
\text { techo (\%) }\end{array}$ & $\begin{array}{c}\text { Alfa de } \\
\text { Cronbach }^{-}\end{array}$ & $\begin{array}{c}\text { ICC medidas } \\
\text { individuales }\end{array}$ \\
\hline Bienestar físico & 5 & 0.1 & 0 & .50 & .54 \\
Bienestar psicológico & 6 & 0 & 11.7 & .83 & .73 \\
Estado de ánimo & 7 & 0 & 2.5 & .81 & .47 \\
Autopercepción & 5 & 0.2 & 7 & .66 & .68 \\
Autonomía & 5 & 0.1 & 17.2 & .86 & .45 \\
Relación con los padres y vida familiar & 6 & 0 & 6.5 & .86 & .78 \\
Amigos y apoyo social & 3 & 0.4 & 6.8 & .85 & .57 \\
Recursos económicos & 3 & 1.4 & 5.4 & .67 & .65 \\
Entorno escolar & 6 & 0.1 & 6.7 & .84 & .22 \\
Aceptación social (Bullying) & 6 & 0.4 & 28.5 & .83 & .57 \\
\hline
\end{tabular}

" T-internacional RASCH. ICC=Coeficiente de correlación intraclase. " De n $=32$ estudiantes.

sanas y enfermas. La puntuaciones obtenidas por los más jóvenes fueron más elevadas en todas las dimensiones respecto de los adolescentes, excepto en la dimensión de aceptación social (Bullying), aspecto ya descrito en un estudio previo ${ }^{30}$.
Por otra parte, las mujeres puntúan peor en la mayoría de dimensiones físicas y psicológicas. Este hecho también se había mostrado en los estudios previos llevados a cabo con el KIDS$\mathrm{CREEN}^{26}$. Algunas de las puntuaciones medias 
han sido ligeramente inferiores al estandarizar la muestra de México con las de la versión Europea. Este hecho podría deberse en parte a las características de la muestra, con mayor porcentaje de estudiantes de más de 15 años. Estudios posteriores que incluyan análisis de función diferencial del ítem podrían resolver esta cuestión.

Se trabajó con la versión para niños y adolescentes KIDSCREEN de 52 ítems, lo que permitiría después también contar con las versiones de menos ítems.

La versión mexicana del KIDSCREEN-52 es un cuestionario adaptado transculturalmente, valido y fiable para medir CVRS en niños y adolescentes de 8 a 18 años y puede ser recomendado como medida genérica de CVRS en niños. Se re-

\section{Referencias}

1. Naughton MJ, Shumaker SA. The case for domains of function in quality of life assessment. Qual Life Res 2003; 12(Supl. 1):73-80.

2. Varni JW, Burwinkle TM, Lane MM. Health-related quality of life measurement in pediatric clinical practice: an appraisal and precept for future research and application. Health Qual Life Outcomes 2005; 3:34.

3. United Nations Population Found (UNFPA). [Internet]. 2013 [acceso 2013 mayo 2]. Disponible en: http://www.unfpa.org/webdav/site/global/shared/ factsheets/One $\% 20$ pager $\% 20$ on $\% 20$ youth $\% 20$ demographics\%20GF.pdf

4. Patrick D. Patient-Reported Outcomes (PROs): An Organizing Tool for Concepts, Measures, and Applications. Quality of Life Newsletter 2003; 31:1-5.

5. Centers for Disease Control and Prevention. Measuring Healthy Days. Atlanta, Georgia: CDC, November 2000. [Internet]. 2000 [acceso 2007 ene 11]. Disponible en: http://www.cdc.gov/hrqol/pdfs/mhd. pdf

6. Hidalgo-Rasmussen C. Calidad de vida relacionada con la salud en los adolecentes. En: Alonso C, editor. Salud y enfermedad del Niño y del Adolescente. Jalisco: Editorial Manual Moderno; 2013. p. 15601563.

7. Helseth S, Misvaer N. Adolescents' perceptions of quality of life: what it is and what matters. J Clin Nurs 2010; 19(9-10):1454-1461.

8. Rajmil L, Alonso J, Berra S, Ravens-Sieberer U, Gosch A, Simeoni MC, Auquier P; KIDSCREEN group. Use of a children questionnaire of healthrelated quality of life (KIDSCREEN) as a measure of needs for health care services. $J$ Adolesc Health 2006; 38(5):511-518. comienda en el futuro estudiar la relevancia en la práctica clínica.

\section{Colaboradores}

CA Hidalgo-Rasmussen trabajó en la concepción, diseño, análisis, interpretación, redacción del artículo, revisión crítica del artículo y aprobación de la versión final. L Rajmil trabajó en el diseño, análisis, interpretación, revisión crítica del artículo y aprobación de la versión final. R Montaño Espinoza trabajó en el análisis, interpretación de los datos, revisión crítica del artículo y aprobación de la versión final.
9. Ravens-Sieberer U, Erhart M, Gosch A, Wille N. European KIDSCREEN Group. Mental health of children and adolescents in 12 European countriesresults from the European KIDSCREEN study. Clin Psychol Psychother 2008; 15(3):154-163.

10. Palacio-Vieira JA, Villalonga-Olives E, Alonso J, Valderas JM, Herdman M, Espallargues M, Berra S Rajmil L. Brief report: The KIDSCREEN follow-up study on Health-related Quality of Life (HRQoL) in Spanish children and adolescents. Pilot test and representativeness. J Adolesc 2010; 33(1):227-231.

11. Silveira MF, Almeida JC, Freire RS, Ferreira RC, Martins AE, Marcopito LF. Quality of life among adolescents: a cross-sectional study using the SF-12 questionnaire. Cien Saude Colet 2013; 18(7):2007-2015.

12. Pane S, Solans M, Gaite L, Serra-Sutton V, Estrada $\mathrm{M}$, Rajmil L. Instrumentos de calidad de vida relacionada con la salud en edad pediátrica Revisión sistemática de la literatura: actualización Barcelona: Agència d'Avaluació de Tecnologia i Recerca Mèdiques; 2006.

13. Rajmil L, Roizen M, Urzúa A, Hidalgo-Rasmussen C, Fernández G, Dapueto JJ. Health-related quality of life measurement in children and adolescents in Ibero-American countries, 2000 to 2010. Value Health 2012; 15(2):312-322

14. Soares AH, Martins AJ, Lopes Mda C, Britto JA, Oliveira CQ, Moreira MC. Quality of life of children and adolescents: a bibliographical review. Cien Saude Colet 2011; 16(7):3197-3206.

15. Berra S, Bustingorry V, Henze C, Diaz M, Rajmil L, Butinof M. Adaptación transcultural del cuestionario KIDSCREEN para medir calidad de vida relacionada con la salud en población argentina de 8 a 18 años. Arch Argent Pediatr 2009; 107(4):307-314. 
16. Aymerich M, Berra S, Guillamon I, Herdman M, Alonso J, Ravens-Sieberer U, Rajmil L. Desarrollo de la versión en español del KIDSCREEN, un cuestionario de calidad de vida para la población infantil y adolescente. Gac Sanit 2005; 19(2):93-102.

17. Ravens-Sieberer U, Gosch A, Rajmil L, Erhart M, Bruil J, Power M, Duer W, Auquier P, Cloetta B, Czemy L, Mazur J, Czimbalmos A, Tountas Y, Hagquist C, Kilroe J; KIDSCREEN Group. The KIDSCREEN-52 quality of life measure for children and adolescents: psychometric results from a cross-cultural survey in 13 European countries. Value Health 2008; 11(4):645-658.

18. The KIDSCREEN Group. Description of the KIDSCREEN instruments. [Internet]. 2004 [acceso 2013 jun 3]. Disponible en: http://www.kidscreen.org/ español/el-proyecto-kidscreen/

19. Guedes D, Guedes J. Translation, cross-cultural adaptation and psychometric properties of the KIDSCREEN-52 for the Brazilian population. Rev paul pediatr 2011; 29(3):364-371.

20. Sepúlveda PR, Molina GT, Molina CR, Martínez NV, González AE, L MG, Montaño E R, HidalgoRasmussen C. Adaptación transcultural y validación de un instrumento de calidad de vida relacionada con la salud en adolescentes chilenos. Rev Med Chil 2013; 141(10):1283-1292.

21. Jaimes-Valencia M, Cabrero R, Flores-Alarcón L, Palacio J. Calidad de vida relacionada con la salud en niños (as) - adolescentes: adaptación cultural del instrumento KIDSCREEN-52 en Colombia. En: Libro de ponencias del X Encuentro Internacional de investigación en Enfermería; 2006; Madrid. p. 75-77.

22. Gaspar T, Matos M. Qualidade de vida em crianças e adolescentes versão portuguesa dos instrumentos KIDSCREEN 52. Lisboa: Aventura Social e Saúde; 2008.

23. Scientific Advisory Comitee of the Medical Outcomes Trust. Assessing health status and qualityof-life instruments: attributes and review criteria. Qual Life Res 2002; 11(3):193-205.
24. The KIDSCREEN Group. KIDSCREEN Translation Manual. [Internet]. 2004 [acceso 2009 nov 17]. Disponible en: http://kidscreen.port4949.net/cms/ sites/kidscreen.port4949.net/files/KIDSCREEN_ Translation_Manual.pdf

25. Valderas JM, Ferrer M, Mendivil J, Garin O, Rajmil L, Herdman M, Alonso J. Scientific Committee on "Patient-Reported Outcomes" of the IRYSS Network. Development of EMPRO: A Tool for the Standardized Assessment of Patient-Reported Outcome Measures. Value Health 2008; 11(4):700-708.

26. Berra S, Tebé C, Esandi ME, Carignano C. Reliability and validity of the KIDSCREEN-52 questionnaire to measure health related quality of life in the 8 to 18 year-old Argentinean population. Arch Argent Pediatr 2013; 111(1):29-36.

27. Lloyd K. Kids' Life and Times: using an Internet survey to measure children's health-related quality of life. Qual Life Res 2011; 20(1):37-44.

28. Nunnally J, Bernstein I. Teoría psicométrica. Brasília, DF: McGraw-Hill; 1995.

29. Faul F, Erdfelder E, Buchner A, Lang AG. Statistical power analyses using $G^{\star}$ Power 3.1: Tests for correlation and regression analyses. Behav Res Methods 2009; 41(4):1149-1160.

30. Analitis F, Klein M, Ravens-Sieberer U, Detmar S, Erhart M, Herdman M, Berra S, Alonso J, Rajmil L; European Kidscreen Group. Being bullied: associated factors in children and adolescents 8 to 18 years old in 11 European countries. Pediatrics 2009; 123(2):569-577.

Artigo apresentado em 17/06/2013

Aprovado em 23/09/2013

Versão final apresentada em 05/10/2013 